\title{
Prognostic Significance of Surgical Margin Status and Gleason Grade at the Positive Surgical Margin in Predicting Biochemical Recurrence After Radical Prostatectomy in a Turkish Patient Cohort
}

\author{
(1) Murat Yavuz Koparal ${ }^{1}$, (1) Tevfik Sinan Sözen², (1) Güven Aslan ${ }^{3}$, (1) Sümer Baltacl ${ }^{4}$, (1) Evren Süer ${ }^{4}$, (1) Talha Müezzinoğlu5, \\ (1) Bülent Akdoğan ${ }^{6}$, (D Levent Türkeri ${ }^{7}$
}

${ }^{1}$ Recep Tayyip Erdoğan University Training and Research Hospital, Clinic of Urology, Rize, Turkey ${ }^{2}$ Gazi University Faculty of Medicine, Department of Urology, Ankara, Turkey ${ }^{3}$ Dokuz Eylül University Faculty of Medicine, Department of Urology, Izmir, Turkey

${ }^{4}$ Ankara University Faculty of Medicine, Department of Urology, Ankara, Turkey

${ }^{5}$ Celal Bayar University Faculty of Medicine, Department of Urology, Manisa, Turkey

6 Hacettepe University Faculty of Medicine, Department of Urology, Ankara, Turkey

${ }^{7}$ Acıbadem University Faculty of Medicine, Department of Urology, Istanbul, Turkey

\begin{abstract}
Objective: To investigate the prognostic role of positive surgical margin (PSM) features in addition to well-defined risk factors in predicting biochemical recurrence (BCR) after radical prostatectomy.

Materials and Methods: This study used the prostate cancer database from the Urooncology Association in Turkey. Clinical, surgical, pathological and follow-up data were recorded from the database. PSM features, including number, location, linear length and Gleason grade (GG) were also recorded. Kaplan-Meier survival analyses were performed to assess differences in BCR-free survival (BCR-FS). In order to identify prognostic factors affecting BCR-FS, univariate and multivariate Cox regression analyses were performed.

Results: The study included 984 patients who met the eligibility criteria. The median follow-up time was 29 (minimum: 6, maximum: 210) months, and BCR was detected in $178(18.1 \%)$ patients. BCR-FS was found to be significantly lower in patients with higher total prostate-specific antigen, higher International Society of Urological Pathology (ISUP) grade, extraprostatic extension (EPE), seminal vesicle invasion, lymphovascular invasion, lymph node involvement, PSM and GG at PSM (PSMGG) $\geq 4$ (log-rank $p<0.001, p<0.001, p<0.001, p<0.001, p<0.001, p<0.001, p<0.001$ and $p=0.005$ ). ISUP grade, EPE and PSM were identified as independent prognostic factors in predicting BCR-FS [Hazard ratio (HR): 1.89, $p=0.035$ and HR: 4.65, $p<0.001$, HR: 1.82, $p=0.030$, HR: 1.77, $p=0.042$, respectively]. Unlike the univariate analysis, in multivariate analysis, PSMGG did not prove to be an independent prognostic factor in predicting BCR-FS.

Conclusion: PSM GG $\geq 4$ was found to be significantly associated with shorter BCR-FS. There is a need for large, randomised prospective studies to clarify the role of PSMGG to be used in nomograms as an independent predictor to determine patients who would benefit from adjuvant radiation therapy.

Keywords: Radical prostatectomy, positive surgical margin, Gleason grade
\end{abstract}

\section{Introduction}

Prostate cancer, the most common newly diagnosed cancer in men, is the second leading cause of cancer-related deaths (1). Biochemical recurrence (BCR), the most common pattern of disease relapse, is seen in nearly $30 \%$ of patients who have undergone radical prostatectomy (RP) (2). Numerous risk factors, including preoperative total prostate-specific antigen (PSA) level, pathological stage, Gleason grade (GG), perineural invasion (PNI), lymphovascular invasion (LVI) and positive surgical margin (PSM) have been identified in predicting BCR. PSM is seen in $10 \%-48 \%$ of patients after RP (3). Moreover, half of all patients with PSM develop BCR (4). The absence of BCR in a significant proportion of patients with PSM necessitates

Cite this article as: Koparal MY, Sözen TS, Aslan G, Baltacı S, Süer E, Müezzinoğlu T, Akdoğan B, Türkeri L. Prognostic Significance of Surgical Margin Status and Gleason Grade at the Positive Surgical Margin in Predicting Biochemical Recurrence After Radical Prostatectomy in a Turkish Patient Cohort. Bull Urooncol 2021;20(1):26-33

Address for Correspondence: Murat Yavuz Koparal, Recep Tayyip Erdoğan University Training and Research Hospital, Clinic of Urology, Rize, Turkey Phone: +90 3122156156 E-mail: drkoparal@yahoo.com ORCID-ID: orcid.org/0000-0002-8347-5727 
the examination of surgical margin features, including number, linear length, location and GG at the site of positive resection margin. This study investigates the prognostic role of surgical margin features in addition to well-defined risk factors in predicting BCR.

\section{Materials and Methods}

\section{Study design}

This study used the prostate cancer database from the Urooncology Association in Turkey, to which participating institutions submit data online. Data obtained from 984 patients who underwent RP with localised and locally advanced prostate cancer and met eligibility criteria for this study (Figure 1). Since our study was a retrospective study using a database, informed consent and ethics committee approval was not obtained.

\section{Data Collection and Definitions}

Clinical (age, preoperative total PSA), surgical (type of operation, lymphadenectomy status), pathological [International Society of Urological Pathology (ISUP) grade, extraprostatic extension $(\mathrm{EPE}), \mathrm{LVI}, \mathrm{PNI}$, seminal vesicle invasion (SVI), surgical margin status, lymph node involvement (LNI)], PSM (number, linear length, location and GG) and follow-up (PSA outcome, BCR status) data were requested and recorded from the prostate cancer database. PSM features including linear length $(<1 \mathrm{~mm}$ and $\geq 1 \mathrm{~mm}$ ), number (single and multiple), location (apex, anterior, posterolateral, bladder neck and seminal vesicle) and GG (1-5) were recorded as submitted in the prostate cancer database. The ISUP grading system as identified in the 2014 ISUP consensus conference was used (5). BCR is defined as PSA $>0.2 \mathrm{ng} / \mathrm{mL}$ after the RP (6). BCR-free survival (BCR-FS) is defined as the time from the date of RP to the date of BCR. In patients without BCR, BCR-FS is defined as the time from the date of RP to the date of the last follow-up visit.

\section{Statistical Analysis}

The normal distribution of continuous variables was evaluated through visual (histogram and probability plots) and analytical (Kolmogorov-Smirnov and Shapiro-Wilk tests) methods. For continuous variables, the statistical difference among the groups

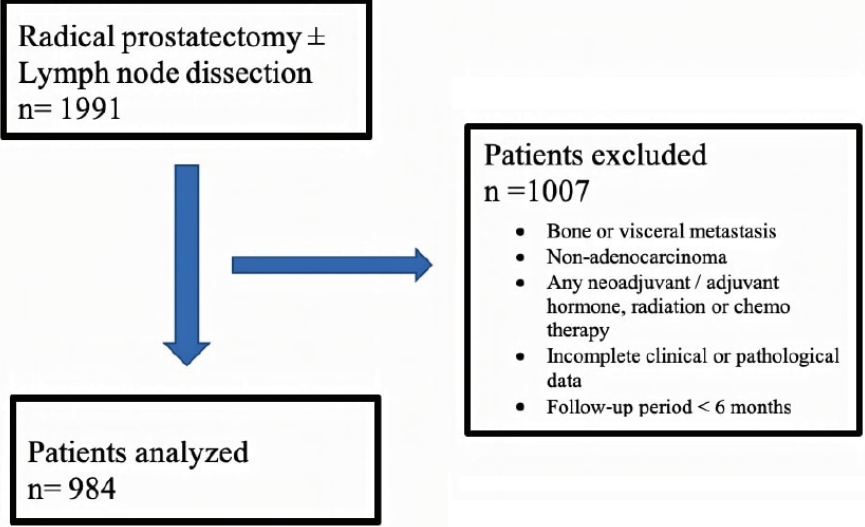

Figure 1. Flow chart of the patients who met study eligibility was determined using the Mann-Whitney $\mathrm{U}$ test. For categorical variables, statistically significant differences among groups were determined using chi-square tests. To assess differences in BCRFS, Kaplan-Meier curves were generated and compared using the log-rank test. Univariate and multivariate Cox regression analyses were performed to identify the prognostic factors affecting BCR-FS. Statistical significance was accepted as $\mathrm{p}<0.05$. IBM SPSS Statistics 15.0 was used for the statistical analysis.

\section{Results}

\section{Clinicopathological Characteristics}

Patients' clinical, surgical and pathological data are summarised in Table 1. BCR was detected in 178 (18.1\%) patients. The median follow-up time for all patients was 29 (6-210) months, and it was significantly higher in the BCR group (36 months vs 28 months). The total PSA was significantly higher in the BCR group ( $8.3 \mathrm{ng} / \mathrm{mL}$ vs, $6.7 \mathrm{ng} / \mathrm{mL} ; \mathrm{p}<0.001)$. Data analysis showed that a higher ISUP grade had a significant relationship with BCR $(p<0.001)$. Moreover, EPE, SVI, LVI and LNI were all significantly higher in the BCR cohort $(p<0.001)$.

\section{Surgical Margin Features}

The PSM was significantly higher in the BCR group $(53.4 \%$ vs $29.5 \% ; p<0.001)$. Surgical margin status was evaluated in terms of surgical margin features, including number, location, linear length and GG at PSM. There was no statistically significant difference between the BCR and disease-free group in terms of number, location and linear length of PSM ( $>>0.05)$. Patients were separated into two groups according to positive surgical margin Gleason grade (PSMGG) as PSMGG $\leq 3$ and PSMGG $\geq 4$. We found a significant relationship between higher PSMGG and BCR $(p=0.043)$. We also analysed the PSMGG status in three groups-downgrade, same and upgrade-according to the difference from the index tumour GG. We found a significant relationship between the downgrade of PSMGG and diseasefree status (Table 2).

\section{Relationship Between BCR-FS and Clinicopathological Features}

The median follow-up period was 28 (6-210) months in the disease-free group. According to Kaplan-Meier survival analysis, BCR-FS was found to be significantly lower in the group with the higher total PSA level, higher ISUP grade, EPE, SVI, LVI and LNI (log-rank $p<0.001)$. Kaplan-Meier survival curves, including 2-year, 5-year and 10-year BCR-FSs, are shown in Figure 2.

\section{Relationship Between BCR-FS and Surgical Margin Features}

In 333 (33.8\%) patients, PSMs were observed. Since PSMGG was not routinely reported in every patient with a PSM, 120 patients were included in the analysis involving PSMGG. According to Kaplan-Meier survival analysis, BCR-FS was found to be significantly higher in the group with a negative surgical margin (NSM) (log-rank $p<0.001)$ (Figure 3a). The NSM group was compared separately from the PSMGG $\leq 3$ and PSMGG $\geq 4$ groups in terms of BCR-FS. It was shown that patients with NSM 


\begin{tabular}{|c|c|c|c|}
\hline & Disease-free & Biochemical recurrence & p-value \\
\hline Age (year) [median (min-max)] & $63(30-83)$ & $63(46-75)$ & 0.334 \\
\hline Total PSA (ng/dL) [median (min-max)] & $6.7(0.82-87.0)$ & $8.3(0.73-64.1)$ & $<0.001$ \\
\hline \multicolumn{4}{|l|}{ Type of operation n (\%) } \\
\hline Open & $617(79.3)$ & $146(86.4)$ & \multirow{3}{*}{0.033} \\
\hline Robotic & $131(16.8)$ & $15(8.9)$ & \\
\hline Laparoscopic & $30(3.9)$ & $8(4.7)$ & \\
\hline \multicolumn{4}{|l|}{ Lymph node dissection $\mathrm{n}(\%)$} \\
\hline Yes & $318(39.5)$ & $97(54.5)$ & \multirow{2}{*}{$<0.001$} \\
\hline No & $488(60.5)$ & $81(45.5)$ & \\
\hline \multicolumn{4}{|l|}{ ISUP grade $\mathbf{n}(\%)$} \\
\hline 1 & $306(38.0)$ & $47(26.4)$ & \multirow{5}{*}{$<0.001$} \\
\hline 2 & $347(43.1)$ & $51(28.7)$ & \\
\hline 3 & $93(11.5)$ & $34(19.1)$ & \\
\hline 4 & $36(4.5)$ & $15(8.4)$ & \\
\hline 5 & $24(3.0)$ & $31(17.4)$ & \\
\hline \multicolumn{4}{|l|}{ Extraprostatic extension n (\%) } \\
\hline Yes & $167(22.1)$ & $65(45.5)$ & \multirow{2}{*}{$<0.001$} \\
\hline No & $590(77.9)$ & $78(54.5)$ & \\
\hline \multicolumn{4}{|l|}{ Seminal vesicle invasion $\mathbf{n}(\%)$} \\
\hline Yes & $49(6.1)$ & $47(26.7)$ & \multirow{2}{*}{$<0.001$} \\
\hline No & $748(93.9)$ & $129(73.3)$ & \\
\hline \multicolumn{4}{|l|}{ Lymphovascular invasion } \\
\hline Yes & $24(3.0)$ & $24(13.8)$ & \multirow{2}{*}{$<0.001$} \\
\hline No & $776(97.0)$ & $150(86.2)$ & \\
\hline \multicolumn{4}{|l|}{ Perineural invasion } \\
\hline Yes & $497(62.3)$ & $110(63.6)$ & \multirow{2}{*}{0.748} \\
\hline No & $301(37.7)$ & $63(36.4)$ & \\
\hline \multicolumn{4}{|l|}{ Lymph node involvement $\mathrm{n}(\%)$} \\
\hline Yes & $13(4.2)$ & $18(18.9)$ & \multirow{2}{*}{$<0.001$} \\
\hline No & $296(95.8)$ & $77(81.1)$ & \\
\hline Follow-up (month) [median (min-max)] & $28(6-210)$ & $36(6-196)$ & $<0.001$ \\
\hline BCR time (month) [median (min-max)] & & $12(6-166)$ & \\
\hline
\end{tabular}

had a significantly better BCR-FS than patients with PSMGG $\geq 4$ ( $\log$-rank $p=0.005)$. However, there was no significant difference between patients with NSM and PSMGG $\leq 3$ in terms of BCR-FS (log-rank $p=0.662$ ). On account of this finding, we combined NSM and PSMGG $\leq 3$ groups into a single cohort, and then compared with PSMGG $\geq 4$ group. According to KaplanMeier survival analysis, BCR-FS was still significantly lower in the PSMGG $\geq 4$ group (Figure 3 b). Kaplan-Meier survival curves, including 2-year and 5-year BCR-FSs, are shown in Figure 3.

\section{Prognostic Factors in Predicting BCR-FS}

Univariate Cox regression analysis indicated that total PSA, ISUP grade, EPE, SVI, LVI, LNI, PSM and PSMGG are significantly associated with BCR-FS $(p<0.001, p<0.001, p<0.001, p<0.001$, $p<0.001, \quad p<0.001, p<0.001$ and $p=0.007$, respectively).
Multivariate Cox regression analysis was performed to identify the independent prognostic factors for predicting BCR. For this purpose, we created two different predictive models. We included total PSA, ISUP grade, EPE, SVI LVI and LNI in both models. In addition to these predictive factors, Model 1 included surgical margin status, and Model 2 included PSMGG. When evaluating PSMGG in multivariate Cox regression analysis, we combined patients with NSM and PSMGG $\leq 3$ into the same group, and then compared them with PSMGG $\geq 4$. In multivariate Cox regression analysis of Model 1, ISUP grade, EPE and PSM were found to be independent prognostic factors in predicting BCR-FS [Hazard ratio (HR): 1.89, $\mathrm{p}=0.035$ and HR: 4.65, $\mathrm{p}<0.001$, HR: 1.82, $\mathrm{p}=0.030$, HR: 1.77, $p=0.042$, respectively]. However, in Model 2 , only ISUP grade was found to be an independent prognostic factor in predicting BCR-FS (HR: 10.04, p<0.001, Table 3). 

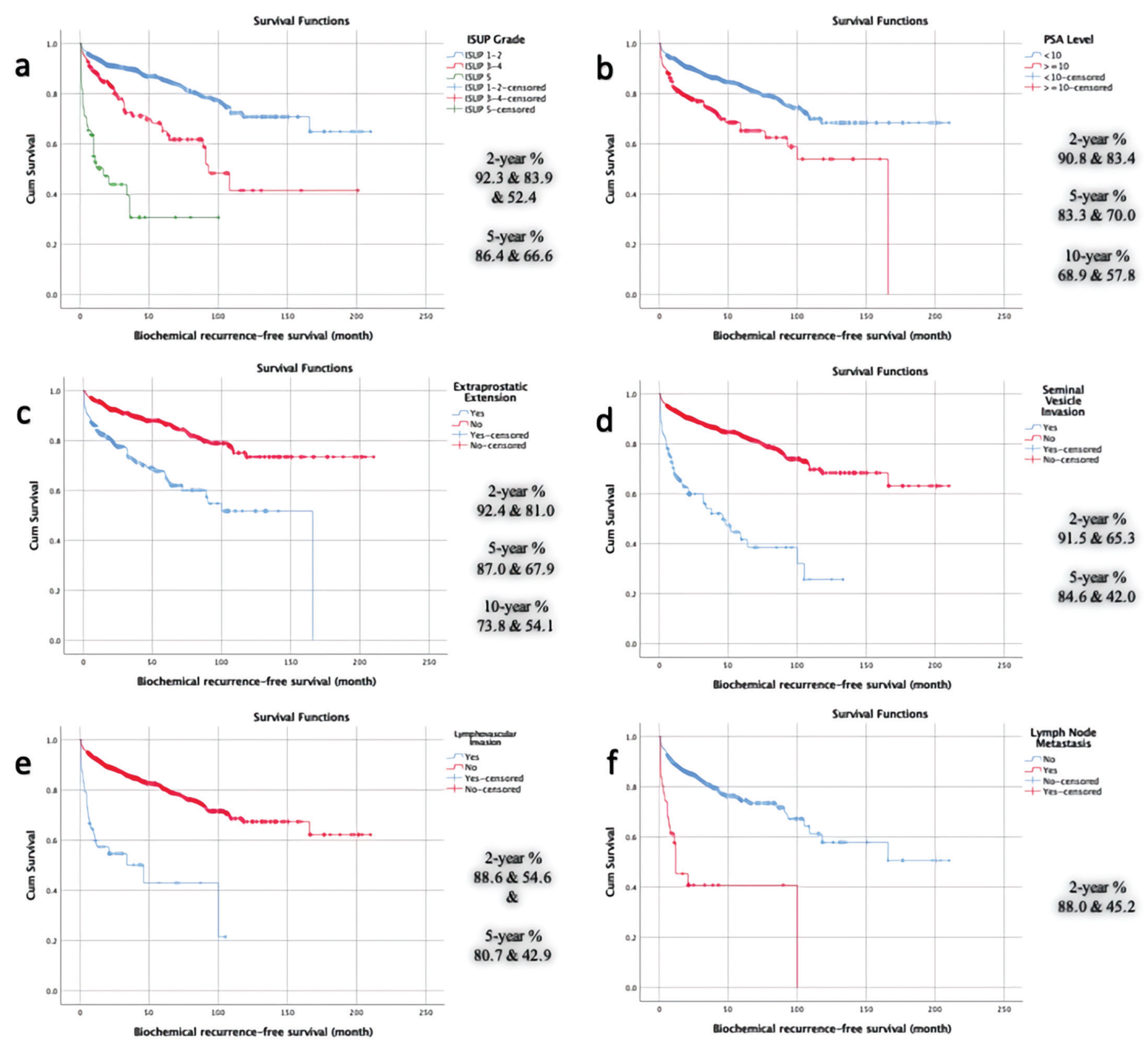

Figure 2. Kaplan-Meier curves in predicting biochemical recurrence-free survival, and 2, 5 and 10-year survival probability, groups categorised in terms of (a) ISUP grade, (b) PSA level, (c) extraprostatic invasion status, (d) seminal vesicle invasion status, (e) lymphovascular invasion status and (f) lymph node involvement status ISUP: International Society of Urological Pathology, PSA: Prostate-specific antigen

\section{Discussion}

Patients with BCR after RP have a worse prognosis. In these patients, the risk of metastatic disease and cancer-related death increases significantly (2). Cancer-specific survival declines significantly in patients with a shorter PSA doubling time and shorter interval to BCR after RP as well as in patients with higher Gleason scores (GSs). A PSM and higher pathologic tumour stage were also found to increase the risk of metastatic disease (7). Prediction of BCR-FS plays an important role in determining which patients would benefit from adjuvant treatment, especially radiation therapy (ART). In a meta-analysis including three randomised-controlled trials (EORTC22911, SWOG8794, ARO96-02/AUO-AP09/95) in which ART was compared with a wait-and-see strategy, metastasis-FS was found to be significantly higher in the ART group (Odds ratio=0.77, $p=0.02$ ). Clinical progression-FS was found to be significantly lower in patients with SVI or PSM (HR=0.73, p=0.0003) (8). In another multi-institutional study, no significant difference was found in terms of metastasis-FS and overall survival when early salvage radiotherapy and ART were compared (92\% vs $91 \%, \mathrm{p}=0.9$, and $89 \%$ vs $92 \%, p=0.9$, respectively) (9). 


\begin{tabular}{|c|c|c|c|}
\hline & Disease-free & $\begin{array}{l}\text { Biochemical } \\
\text { recurrence }\end{array}$ & p-value \\
\hline \multicolumn{4}{|c|}{ Surgical margin status n (\%) } \\
\hline Positive & $238(29.5)$ & $95(53.4)$ & \multirow{2}{*}{$<0.001$} \\
\hline Negative & $568(70.5)$ & $83(46.6)$ & \\
\hline \multicolumn{4}{|c|}{ Number of PSM $\mathbf{n}(\%)$} \\
\hline Single & $151(77.0)$ & $52(74.3)$ & \multirow{2}{*}{0.642} \\
\hline Multiple & $45(23.0)$ & $18(25.7)$ & \\
\hline \multicolumn{4}{|c|}{ Location of PSM n (\%)* } \\
\hline Apex & $47(31.1)$ & $15(28.8)$ & \multirow{5}{*}{0.950} \\
\hline Anterior & $23(15.2)$ & $10(19.2)$ & \\
\hline Posterolateral & $71(47.0)$ & $24(46.2)$ & \\
\hline Bladder neck & $8(5.2)$ & $2(3.8)$ & \\
\hline Seminal vesicle & $2(1.3)$ & $1(1.9)$ & \\
\hline \multicolumn{4}{|c|}{ Linear length of PSM $\mathbf{n}(\%)$} \\
\hline$<1 \mathrm{~mm}$ & $39(37.5)$ & $7(31.8)$ & \multirow{2}{*}{0.615} \\
\hline$\geq 1 \mathrm{~mm}$ & $65(62.2)$ & $15(68.2)$ & \\
\hline \multicolumn{4}{|c|}{ Gleason grade of PSM n (\%) } \\
\hline 1 & $7(7.6)$ & 0 & \multirow{5}{*}{ *0.043 } \\
\hline 2 & $16(17.4)$ & 0 & \\
\hline 3 & $45(48.9)$ & $9(50.0)$ & \\
\hline 4 & $18(19.6)$ & $6(33.3)$ & \\
\hline 5 & $6(6.5)$ & $3(16.7)$ & \\
\hline \multicolumn{4}{|c|}{ Comparison of PSMGG with index tumor GG n (\%) } \\
\hline Downgrade & $32(34.8)$ & $1(5.6)$ & \multirow{3}{*}{0.042} \\
\hline Same & $35(38.0)$ & $9(50.0)$ & \\
\hline Upgrade & $25(27.2)$ & $8(44.4)$ & \\
\hline
\end{tabular}

Preoperative PSA, pathological GS, EPE, SVI, PSM and LNI are included in nomograms as independent predictive factors for BCR (10). Kattan et al. (11) created the first nomogram in 1999, including these predictors, and external validations of this nomogram have been performed subsequently $(12,13)$. In our study, we found a statistically significant relationship between high preoperative PSA, high pathological ISUP grade, EPE, SVI, PSM, LVI and LNI and a shorter BCR-FS in univariate analysis. In a meta-analysis evaluating the prognostic significance of six clinicopathological features including PSM, EPE, SVI, LVI, $\mathrm{PNI}$ and $\mathrm{LNI}$, all these factors were found to be statistically significant for BCR-FS (HR: 1.79, $\mathrm{p}<0.001$; HR: 2.03, $\mathrm{p}<0.001$; HR: 1.97; $p<0.001$; HR: 1.85, $p<0.001$; HR: 1.59, $p<0.001$; HR: $1.88, p<0.001$, respectively) (14). LVI and PNI are not involved in current prostate cancer nomograms. The prognostic role of $\mathrm{PNI}$ in predicting $\mathrm{BCR}$ is controversial. In a large multicentre study, Kraus et al. (15) found that PNI was not an independent predictor for BCR. However, it can be an indicator of unfavourable histology, such as high G. In our study, we found no statistically significant relationship between PNI and BCR. However, LVI was found to have a significant association with a higher BCR risk in a meta-analysis evaluating its prognostic value (16). We found a statistically significant relationship between high total PSA, high ISUP grade, PSM, EPE, SVI, LVI and LVI and BCR. Kaplan-Meier analyses also showed shorter BCR-FS in these groups. However, $\mathrm{PNI}$ was not statistically associated with $\mathrm{BCR}$.

PSM is a well-known predictor for BCR $(17,18,19)$. However, there is limited evidence about PSM subgroups, including number, location, linear length and GG/GS. Apical and posterolateral prostate were the most common locations in terms of PSM (20). Although the prognostic effect of location and number of PSM on BCR is controversial $(20,21,22,23)$, the relationship between both extended positive surgical margin linear length (PSMLL) and higher PSMGG/SMGS with BCR has been previously reported. Mainly PSMLL $\geq 3 \mathrm{~mm}$ and PSMGG $\geq 4$ have been shown to be the most prominent factors in predicting $B C R(24,25,26,27,28,29)$. Unlike most studies, we grouped PSMLL as $<1 \mathrm{~mm}$ and $\geq 1 \mathrm{~mm}$ since the database was designed in this way. We found no significant relationship between number, linear length and location of PSM and BCR. However, we found a statistically significant relationship between PSMGG and BCR. PSMGG $\geq 4$ was found to be associated with a significantly shorter BCR-FS. Iremashvili et al. (27) designed a study in which they provided different "PSM GS" definitions (GS at the margin, high-grade Gleason pattern present at the margin, predominantly high-grade GS at the margin, GS at the margin higher than overall GS and GS at the margin lower than overall GS) and were used to evaluate BCR-FS. Their results showed that all the definitions of GS at PSM were independently associated with the risk of BCR. It was stated that a "high-grade Gleason pattern at a PSM" could be used as the most useful definition since it provided at least as much prognostic benefit as the others. We also found a statistically significant relationship between a lower PSMGG than the overall GG and disease-free status.

We developed two predictive models by using total PSA, ISUP grade, EPE, SVI, LVI, LNI, surgical margin status and PSMGG. In Model 1, which included surgical margin status instead of PSMGG, ISUP grade, EPE and surgical margin status were found to be independent prognostic factors in predicting BCRFS. However, in Model 2, which included PSMGG instead of surgical margin status, only ISUP grade was found to be an independent prognostic factor in predicting BCR-FS. In Model 1 , the expected results were determined in accordance with the current literature except for the SVI $(10,12,14)$. Since there were only 120 patients with PSMGG data, we had to evaluate patients with NSM and PSMGG $\leq 3$, which have similar BCR-FS, in the same group against patients with $P S M G G \geq 4$. A possible explanation for the unexpected result in Model 2 may have been due to the smaller number and the shorter follow-up time of the patients who have the PSMGG data in contrast to previous studies which identified the independent prognostic role of PSMGG in predicting BCR-FS $(24,25,26,27,30)$.

\section{Study Limitations}

The study is retrospective. There were limited data, including LND and PSM features. Since the type of LND was not always recorded, they were all evaluated in the same group. Not performing LND in almost half of the patients led to these patients not being able to be included in the multivariate 
analyses. Although the study included 333 patients with PSM, PSMGG data were only available for 120 patients. Since the PSMGG data had been reported relatively recently, these patients

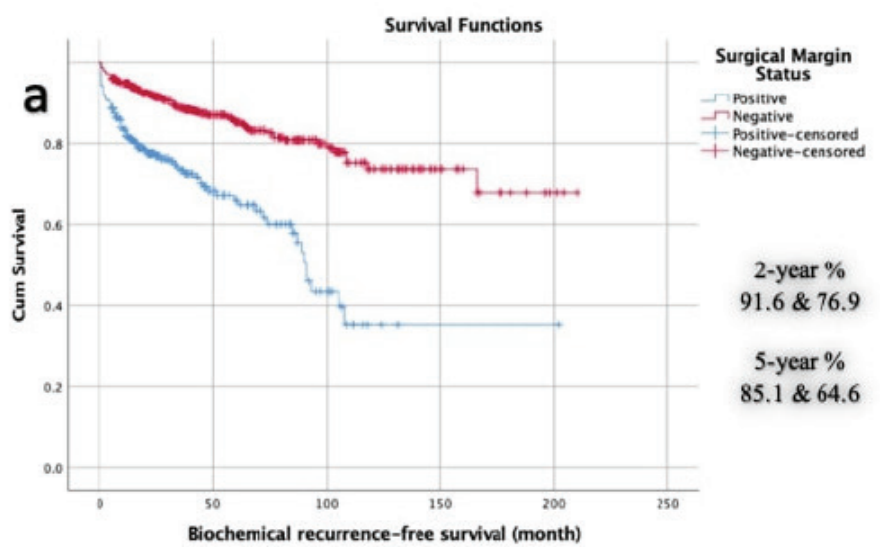

had a shorter follow-up. We thought that the lack of PSM data caused less accurate results in multivariate analysis, which included PSMGG. Moreover, there might have been a difference

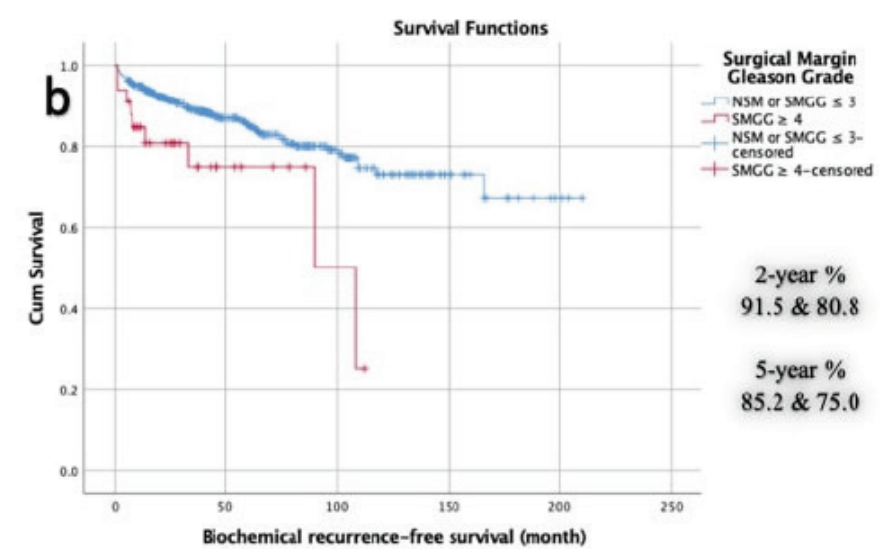

Figure 3. Kaplan-Meier curves in predicting biochemical recurrence-free survival, and 2- and 5-year survival probability, groups categorised in terms of (a) surgical margin status and (b) positive surgical margin Gleason grade

\begin{tabular}{|c|c|c|c|c|c|c|c|c|c|}
\hline & \multicolumn{3}{|c|}{ Univariate analysis } & \multicolumn{3}{|c|}{$\begin{array}{l}\text { Multivariate analysis } \\
\text { Model } 1^{*}\end{array}$} & \multicolumn{3}{|c|}{$\begin{array}{l}\text { Multivariate analysis } \\
\text { Model } 2^{* *}\end{array}$} \\
\hline & $\mathrm{Cl} 95 \%$ & HR & p-value & $\mathrm{Cl} 95 \%$ & HR & p-value & Cl 95\% & HR & p-value \\
\hline \multicolumn{10}{|l|}{ Total PSA } \\
\hline$<10$ & \multirow{2}{*}{$1.62-3.01$} & 1.00 (ref.) & \multirow{2}{*}{$<0.001$} & & & & & & \\
\hline$\geq 10$ & & 2.21 & & & & & & & \\
\hline \multicolumn{10}{|l|}{ ISUP grade } \\
\hline $1-2$ & & 1.00 (ref.) & & & 1.00 (ref.) & & & 1.00 (ref.) & \\
\hline $3-4$ & $1.70-3.39$ & 2.40 & $<0.001$ & $1.04-3.42$ & 1.89 & 0.035 & & & \\
\hline 5 & $5.62-12.8$ & 8.50 & $<0.001$ & $2.08-10.39$ & 4.65 & $<0.001$ & $3.35-30.02$ & 10.04 & $<0.001$ \\
\hline \multicolumn{10}{|c|}{ Extraprostatic extension } \\
\hline No & \multirow{2}{*}{$2.10-4.06$} & 1.00 (ref.) & \multirow{2}{*}{$<0.001$} & \multirow{2}{*}{$1.06-3.12$} & 1.00 (ref.) & \multirow{2}{*}{0.030} & & & \\
\hline Yes & & 2.92 & & & 1.82 & & & & \\
\hline \multicolumn{10}{|c|}{ Lymphovascular invasion } \\
\hline No & \multirow{2}{*}{$2.93-6.98$} & 1.00 (ref.) & \multirow{2}{*}{$<0.001$} & & & & & & \\
\hline Yes & & 4.52 & & & & & & & \\
\hline \multicolumn{10}{|c|}{ Seminal vesicle invasion } \\
\hline No & \multirow{2}{*}{$3.13-6.13$} & 1.00 (ref.) & \multirow{2}{*}{$<0.001$} & & & & & & \\
\hline Yes & & 4.38 & & & & & & & \\
\hline \multicolumn{10}{|c|}{ Lymph node involvement } \\
\hline No & \multirow{2}{*}{$2.70-7.68$} & 1.00 (ref.) & \multirow{2}{*}{$<0.001$} & & & & & & \\
\hline Yes & & 4.55 & & & & & & & \\
\hline \multicolumn{10}{|c|}{ Surgical margin status* } \\
\hline Negative & \multirow{2}{*}{$2.2-4.02$} & 1.00 (ref.) & \multirow{2}{*}{$<0.001$} & \multirow{2}{*}{$1.02-3.10$} & 1.00 (ref.) & \multirow{2}{*}{$<0.042$} & & & \\
\hline Positive & & 2.98 & & & 1.77 & & & & \\
\hline \multicolumn{10}{|l|}{ PSMGG** } \\
\hline NSM or PSMGG $\leq 3$ & \multirow{2}{*}{$1.28-5.06$} & 1.00 (ref.) & \multirow{2}{*}{0.007} & & & & & & \\
\hline PSMGG $\geq 4$ & & 2.55 & & & & & & & \\
\hline
\end{tabular}


in pathologic interpretation, especially when evaluating PSM features, due to the lack of central pathologic review. We also used a cut-off value of $1 \mathrm{~mm}$ for PSM linear length, since it was categorised in the database as such. Continuous linear length data could produce more accurate results.

\section{Conclusion}

The present study shows that ISUP grade, EPE and PSM as independent prognostic factors in predicting BCR-FS. Although BCR-FS was significantly shorter in the patients with PSMGG $\geq 4$, in multivariate analysis, PSMGG was not found as an independent prognostic factor in predicting BCR-FS. There is a need for large, randomised prospective studies to identify the role of PSMGG to be used in nomograms as an independent predictor in order to determine patients who would benefit from ART.

\section{Acknowledgements}

Publication: The results of the study were not published in full or in part in form of abstracts.

Contribution: There is an other contributor who may not be listed as authors: Saadettin Eskiçorapçı, Acıbadem University Faculty of Medicine, Department of Urology, İstanbul, Turkey, Çağ Çal, Ege University Faculty of Medicine, Department of Urology, İzmir, Turkey, Volkan İzol, Çukurova University Faculty of Medicine, Department of Urology, Adana, Turkey.

Conflict of Interest: No conflict of interest was declared by the authors.

Financial Disclosure: The authors declared that this study received no financial support.

Ethics

Ethics Committee Approval: Since our study was a retrospective study using a database, informed consent and ethics committee approval was not obtained.

Informed Consent: Retrospective study.

Peer-review: Externally and internally peer-reviewed.

\section{Authorship Contributions}

Concept: T.S.S., L.T., Design: T.S.S., L.T., Data Collection or Processing: G.A., S.B., E.S., T.M., B.A., Analysis or Interpretation: M.Y.K., Literature Search: M.Y.K., Writing: M.Y.K., T.S.S.

\section{References}

1. Siegel RL, Miller KD, Jemal A. Cancer statistics, 2019. CA Cancer J Clin 2019;69:7-34.

2. Isbarn $\mathrm{H}$, Wanner M, Salomon $\mathrm{G}$, et al. Long-term data on the survival of patients with prostate cancer treated with radical prostatectomy in the prostate-specific antigen era. BJU Int 2010;106:37-43.

3. Eastham JA, Kattan MW, Riedel E, et al. Variations among individual surgeons in the rate of positive surgical margins in radical prostatectomy specimens. J Urol 2003;170(6 Pt 1):2292-2295.

4. Wright JL, Dalkin BL, True LD, et al. Positive surgical margins at radical prostatectomy predict prostate cancer specific mortality. J Urol 2010;183:2213-2218.

5. Epstein Jl, Egevad L, Amin MB, et al. The 2014 International Society of Urological Pathology (ISUP) consensus conference on Gleason grading of prostatic carcinoma. Am J Surg Pathol 2016;40:244-252.
6. Moul JW. Prostate specific antigen only progression of prostate cancer. J Urol 2000;163:1632-1642.

7. Van den Broeck T, van den Bergh RC, Arfi N, et al. Prognostic value of biochemical recurrence following treatment with curative intent for prostate cancer: a systematic review. Eur Urol 2019;75:967-987.

8. Shaikh MP, Alite F, Wu MJ, et al. Adjuvant Radiotherapy Versus Waitand-See Strategy for Pathologic T3 or Margin-Positive Prostate Cancer. Am J Clin Oncol 2018;41:730-738.

9. Fossati N, Karnes RJ, Boorjian SA, et al. Long-term impact of adjuvant versus early salvage radiation therapy in pT3N0 prostate cancer patients treated with radical prostatectomy: results from a multiinstitutional series. Eur Urol 2017;71:886-893.

10. Chun FKH, Karakiewicz PI, Briganti A, et al. Prostate cancer nomograms: an update. Eur Urol 2006;50:914-926.

11. Kattan MW, Wheeler TM, Scardino PT. Postoperative nomogram for disease recurrence after radical prostatectomy for prostate cancer. J Clin Oncol 1999;17:1499-1507.

12. Ondracek RP, Kattan MW, Murekeyisoni C, et al. Validation of the Kattan nomogram for prostate cancer recurrence after radical prostatectomy. J Natl Compr Canc Netw 2016;14:1395-1401.

13. Kattan MW, Vickers AJ, Yu C, et al. Preoperative and postoperative nomograms incorporating surgeon experience for clinically localized prostate cancer. Cancer 2009;115:1005-1010.

14. Liu H, Zhou H, Yan $L$, et al. Prognostic significance of six clinicopathological features for biochemical recurrence after radical prostatectomy: a systematic review and meta-analysis. Oncotarget 2017;9:32238-32249.

15. Kraus RD, Barsky A, Ji L, et al. The perineural invasion paradox: Is perineural invasion an independent prognostic indicator of biochemical recurrence risk in patients with pT2N0R0 prostate cancer? A multi-institutional study. Adv Radiat Oncol 2019;4:96-102.

16. Jiang W, Zhang L, Wu B, et al. The impact of lymphovascular invasion in patients with prostate cancer following radical prostatectomy and its association with their clinicopathological features: An updated PRISMA-compliant systematic review and meta-analysis. Medicine (Baltimore) 2018;97:e13537.

17. Karakiewicz PI, Eastham JA, Graefen M, et al. Prognostic impact of positive surgical margins in surgically treated prostate cancer: multi-institutional assessment of 5831 patients. Urology 2005;66:1245-1250.

18. Swindle P, Eastham JA, Ohori $M$, et al. Do margins matter? The prognostic significance of positive surgical margins in radical prostatectomy specimens. J Urol 2005;174:903-907.

19. Zhang L, Wu B, Zha Z, et al. Positive surgical margin is associated with biochemical recurrence risk following radical prostatectomy: a meta-analysis from high-quality retrospective cohort studies. World J Surg Oncol 2018;16:124.

20. Fleshner NE, Evans A, Chadwick K, et al. Clinical significance of the positive surgical margin based upon location, grade, and stage. Urol Oncol 2010;28:197-204.

21. Blute ML, Bostwick DG, Bergstralh EJ, et al. Anatomic site-specific positive margins in organconfined prostate cancer and its impact on outcome after radical prostatectomy. Urology 1997;50:733-739.

22. Pettus JA, Weight C], Thompson Cl, et al. Biochemical failure in men following radical retropubic prostatectomy: impact of surgical margin status and location. J Urol 2004;172:129-132.

23. Sammon JD, Trinh QD, Sukumar $S$, et al. Risk factors for biochemical recurrence following radical perineal prostatectomy in a large contemporary series: a detailed assessment of margin extent and location. Urol Oncol 2013;31:1470-1476.

24. Kates M, Sopko NA, Han M, et al. Importance of reporting the Gleason score at the positive surgical margin site: analysis of 4,082 consecutive radical prostatectomy cases. J Urol 2016;195:337-342.

25. Chapin BF, Nguyen JN, Achim MF, et al. Positive margin length and highest Gleason grade of tumor at the margin predict for 
biochemical recurrence after radical prostatectomy in patients with organ-confined prostate cancer. Prostate Cancer Prostatic Dis 2018;21:221-227.

26. Preisser $F$, Coxilha $G$, Heinze $A$, et al. Impact of positive surgical margin length and Gleason grade at the margin on biochemical recurrence in patients with organ-confined prostate cancer. Prostate 2019;79:1832-1836.

27. Iremashvili V, Pelaez L, Jorda M, et al. A Comprehensive Analysis of the Association Between Gleason Score at a Positive Surgical Margin and the Risk of Biochemical Recurrence After Radical Prostatectomy. Am J Surg Pathol 2019;43:369-373.
28. Servoll E, Vlatkovic L, Sæeter T, et al. The length of a positive surgical margin is of prognostic significance in patients with clinically localized prostate cancer treated with radical prostatectomy. Urol Int 2014;93:289-295.

29. Koskas Y, Lannes F, Branger N, et al. Extent of positive surgical margins following radical prostatectomy: impact on biochemical recurrence with long-term follow-up. BMC Urol 2019;19:37.

30. Savdie R, Horvath LG, Benito RP, et al. High Gleason grade carcinoma at a positive surgical margin predicts biochemical failure after radical prostatectomy and may guide adjuvant radiotherapy. BJU Int 2012;109:1794-1800. 